\section{Der Arzt im humanitären Dienst: Navigation auf dem Ozean der Bedürfnisse}

\author{
M. Michael
}

Der Krieg - denn darum geht es hier - limitiert sich schon lange nicht mehr darauf, junge Männer in Uniform in Krüppel oder Leichen zu verwandeln. Dies tut er zwar immer noch, wenngleich heute die meisten Opfer von Waffengewalt der Zivilbevölkerung angehören. Dazu, wie einstmals die Pest, erheben längst totgeglaubte oder gebannte Ungeheuer ihr Haupt: Infektionskrankheiten wie Tuberkulose und Poliomyelitis breiten sich wieder hemmungslos aus. Selbst relativ harmlos geglaubte Masern- und Durchfallerkrankungen zeigen durch eine hohe Sterberate wieder ihr mörderisches Potential, hervorgerufen durch Mangel an Verhütung, schlechte Hygiene, späte oder keine Behandlung und einen geschwächten Allgemeinzustand der Erkrankten. Nicht genug damit. Der oft totale Zusammenbruch der Gesundheitsversorgung in langjährigen Kriegen (Jahrzehnte sind keine Seltenheit) führt dazu, dass nicht einmal mehr «alltägliche» Gesundheitsbedürfnisse gedeckt sind: kindliche und mütterliche Sterberaten schnellen hoch, Chronischkranke sowie Patienten mit Malaria oder Appendizitis erliegen ihren Krankheiten. Gewisse Bevölkerungsgruppen sind vermehrten Risiken ausgesetzt, zum Beispiel von ihrer Heimat Vertriebene und Kriegsgefangene. Letztere sind dazu noch jeglicher Freiheitsgrade beraubt, sich gegen Gesundheitsrisiken zu schützen.

Dies ist der Ozean der Gesundheitsbedürfnisse. Wer sich draufwagt, muss mit Schwierigkeiten rechnen. Der Staat ist oft geschwächt oder inexistent und jedenfalls gilt: Gesundheit ist nicht mehr auf der Agenda. Angehörige der wichtigen Berufsstände sind emigriert oder tot. Geld ist auch keines da, weder im Gesundheitsministerium (falls es noch existiert) noch in der einzelnen Haushaltskasse. Im schlimmsten Fall ist die Zivilbevölkerung und ihre Gesundheitsversorgung eine Zielscheibe der Kriegführenden. Kurz: es ist Krieg.

Korrespondenz:

Dr. med. Markus Michael

IKRK

19, avenue de la Paix

CH-1202 Genf
Wer ist ein Opfer des Krieges? Der Krieg geht nicht mehr über einen Ort wie Solferino hinweg und nach der Schlacht wird, wenn es gut geht, einem Häufchen kriegsverletzter Soldaten in einer Kirche die Chance gegeben, die Aufmerksamkeit des Geschäftreisenden Henri Dunant zu erregen. Auch ohne Schlachten fordert der Krieg Opfer, heute sogar deren Mehrzahl.

Die Ärztin und der Arzt, die sich ein Herz fassen und - beispielsweise, um bei Solferino zu bleiben mit dem IKRK in den Krieg ziehen, nehmen nicht nur persönliche Risiken in Kauf, sondern riskieren auch, sich auf dem Ozean der Bedürfnisse zu verlieren. Ist die Möglichkeit gegeben, sich wie damals um Kriegsverletzte zu kümmern, ist es für eine professionelle Organisation möglich, ein Krankenhaus auf die Beine zu stellen. Kaum steht es, ist die Schlacht vorbei, hingegen sorgen Verkehrsunfälle und schwierige Geburten für ausreichend Arbeit, da ja sonst kein Krankenhaus mehr funktioniert. Zusammenpacken und Weiter- oder Weggehen wird schwierig. Oder, im umgekehrten Fall, wenn es einmal möglich ist, für eine bestimmte Bevölkerung eine medizinische Basisversorgung zu gewährleisten: Wohin mit dem Kind, welchem man eine Pneumonie diagnostiziert hat? Es ist oft kein Krankenhaus mehr da. HIV-Gesundheitserziehung wäre auch eine Priorität und Weiterführung der Bekämpfung der Malaria ... Was tun?

Patentlösungen gibt es keine. Aber wie der Ozean durch Längen- und Breitengrade unterteilt ist und es Messinstrumente gibt, sich daran zu orientieren, ist es möglich, auf ebenso künstliche Weise sich auf dem Ozean der Bedürfnisse zu orientieren. Die - konstanteren - Längengrade stellen die Rechte der Kriegsopfer dar, und um sich daran zu orientieren, gibt es legale Instrumente, welche zum Beispiel in der Genfer Konvention gegeben sind. Kolleginnen und Kollegen, die mit dem IKRK arbeiten, haben das Privileg - und die Herausforderung - dank einem professionellen juristischen Navigationsteam, sich zwischen den Längengraden nicht zu verlieren. Herausforderung, weil das Manövrieren gemäss Prinzipien von Neutralität und Impartialität (um nur diese zu nennen) eine humanitäre Diplomatie erfordert, die nicht nur die Rechte der Opfer, sondern auch die Verteidigung eben dieser Prinzipien einschliesst. Realpolitik erfordert, dass Entscheidungen nicht nur nach gesundheitspolitischen Grundsätzen getroffen werden können. Für Kolleginnen und Kollegen, die nicht im öffentlichen Gesundheitsdienst tätig sind, ist dieser Zwiespalt eventuell neu.

Ändern tut sich das Klima mehr, wenn Breitengrade überquert werden, womit wir die Gesundheitsbedürfnisse meinen. Diesmal müssen sich die Kolleginnen und Kollegen selber auskennen. Das Bild der endemischen Krankheiten ist in jedem Land verschieden, vom Balkan bis in den Kongo. Je nach dem Grad des Zerfalls der Gesundheitsversorgung präsentiert sich eine ganze Palette von Bedürfnissen, die nicht mehr gedeckt sind. Darauf pfropfen sich dann, wie eingangs erwähnt, kriegsbedingte Pathologien. 
Allerdings wird auch hier die Aufgabe nicht leichtgemacht: Daten über den Gesundheitszustand der Bevölkerung sind schon lange nicht mehr gesammelt worden oder es werden bewusst falsche in Umlauf gesetzt. Die Bevölkerung als Hauptleidtragende ist oft entrechtet und hat daher Mühe, Bedürfnisse zu artikulieren. Die Sicherheitslage erlaubt Mitarbeitern von Hilfsorganisationen oft nicht, die Situation vor Ort zu erfassen. Um sich unter diesen Umständen zu orientieren und Hilfsmassnahmen einzuleiten, gibt es jedoch viele Instrumente, welche gewissermassen vom sicheren Festland mitgenommen werden können: spezielle Instrumente der Sozial- und Präventivmedizin und allgemeinere Instrumente der Entwicklungszusammenarbeit. Womit nicht gesagt wird, dass im Krieg Raum für Entwicklung ist, wohl aber Raum für die Anwendung einiger ihrer Prinzipien. Wer sich voller Tatendrang nur mit dem Notfallkoffer auf den Ozean wagt, vergisst oftmals, diese Instrumente mitzunehmen oder sie unterwegs sich anzueignen. Grundbegriffe der Epidemiologie zum Beispiel, oder eine Matrix, welche das Auswählen von Gesundheitsbedürfnissen erleichtert, welche in Angriff zu nehmen sind, je nach ihrem Schweregrad und möglicher Angriffsfläche. Andere Instrumente aus der Entwicklungszusammenarbeit sind partizipative Methoden für Entscheidungsprozesse und gezielte Förderung von Integration. Wenn all diese Navigationshilfen voll ausgeschöpft werden, kann die Arbeit lohnend sein, Unbilden zum Trotz.

Nur eben: die Wetterlage auf diesem Ozean ist unbeständig und leicht kann man Schiffbruch erleiden. Die Kunst besteht darin, sich in den Längen- und Breitengraden auszukennen, das Ziel - die Bedürfnisse der Kriegsopfer - nicht aus den Augen zu verlieren und Kurswechsel bewusst vorzunehmen. Solange Kriege andauern, gibt es viel zu tun, nicht nur für Seglerinnen und Segler oder die es werden wollen, sondern auch für den Navigator, der diesen Ozean besser erforschen will.

PS. Mut hat, wer's trotzdem wagt: http://www.icrc.org. 